\title{
PRESSURE MODERATION AND EFFECTIVE PRESSURE IN NAVIER-STOKES FLOWS
}

\author{
CHUONG V. TRAN AND XINWEI YU
}

\begin{abstract}
We study the Cauchy problem of the Navier-Stokes equations by both semi-analytic and classical energy methods. The former approach provides a physical picture of how viscous effects may or may not be able to suppress singularity development. In the latter approach, we examine the pressure term that drives the dynamics of the velocity norms $\|u\|_{L^{q}}$, for $q \geq 3$. A key idea behind this investigation is due to the fact that the pressure $p$ in this term is determined up to a function of both space and $|u|$, say $\mathcal{P}(x,|u|)$, which may assume relatively broad forms. This allows us to use $\mathcal{P}$ as a pressure moderator in the evolution equation for $\|u\|_{L^{q}}$, whereby optimal regularity criteria can be sought by varying $\mathcal{P}$ within its admissible classes. New regularity criteria are derived with and without making use of the moderator. The results obtained in the absence of the moderator feature some improvement over existing criteria in the literature. Several criteria are derived in terms of the moderated (effective) pressure $p+\mathcal{P}$. A simple moderation scheme and the plausibility of the present approach to the problem of Navier-Stokes regularity are discussed.
\end{abstract}

\section{INTRODUCTION}

We study the Cauchy problem of the Navier-Stokes system

$$
\begin{aligned}
\frac{\partial u}{\partial t}+(u \cdot \nabla) u+\nabla p & =\Delta u, \\
\nabla \cdot u & =0, \\
u(x, 0) & =u_{0}(x),
\end{aligned}
$$

where $u$ is the fluid velocity, $p$ is the pressure and the kinematic viscosity coefficient has been set to unity for convenience. The fluid is assumed to fill all $\mathbb{R}^{3}$ with the usual condition of sufficient decay at infinity. The initial velocity field $u_{0}(x)$ is incompressible and sufficiently regular. Given such an initial velocity, it is well known that the solution remains regular at least up to some time $t=T$, which depends on $u_{0}(x)$. The question is whether or not regularity persists beyond $T$, particularly up to all $t \geq T$ (global regularity). Decades of active research on this issue since Leray's seminal studies in the 1930s have resulted in a rich literature [1-35]. Early studies by Prodi [24], Serrin [26] and Ladyzhenskaya [22] found that regularity (up to some time $t$ ) is guaranteed provided that $\int_{0}^{t}\|u\|_{L^{q}}^{2 q /(q-3)} \mathrm{d} \tau<\infty$, for $q>3$. Here $\|\cdot\|_{L^{q}}$ denotes the usual norm in the Lebesque spaces $L^{q}$. Recently, Escauriaza, Seregin and Sverák [14] have extended this criterion to $\operatorname{esssup}_{(0, t)}\|u\|_{L^{3}}<\infty$, for the limiting case $q=3$. On the basis of these results, various criteria expressible in terms of the pressure $p$ and its gradient $\nabla p$ have been derived by a number of authors $[3,6,8,33]$. One of the main results along this line of research is the criterion $\int_{0}^{t}\|p\|_{L^{\alpha}}^{2 \alpha /(2 \alpha-3)} \mathrm{d} \tau<\infty$, for $\alpha \in(3 / 2, \infty)$, representing some moderate improvement of the Prodi-Serrin-Ladyzhenskaya result (due to the

Date: June 18, 2015.

2000 Mathematics Subject Classification. 76D03,76D05.

Key words and phrases. Navier-Stokes equations, Pressure moderation, Effective pressure, Global regularity. 
relation $\|p\|_{L^{\alpha}} \leq c\|u\|_{L^{2 \alpha}}^{2}$, where $c$ is a constant and $\left.1<\alpha<\infty\right)$. This means that a loss of regularity would require not only $|u| \rightarrow \infty$ but also $|p| \rightarrow \infty$. From a quite different approach, Seregin and Sverák [25] proved that no singularities can develop if $p$ is bounded from below. The theory of Navier-Stokes regularity has also been enriched by a number of criteria expressible in terms of one component of $u$ or $\nabla u[5,7,20,21,32,35]$, the velocity direction $u /|u|[9,23,31]$ or vorticity direction $\omega /|\omega|[1,2,10,17,34]$.

The vast wealth of regularity criteria in the literature, together with the slow theoretical progress on the problem, appears to have given the impression that viscous effects are not adequate to regularise the nonlinear dynamics. This impression is strengthened by the critical nature of the criterion $\operatorname{esssup}_{(0, t)}\|u\|_{L^{3}}<\infty$, suggesting that further improvement may not be possible, at least by existing methods. On the contrary, the notion of momentum blow-up is highly counter-intuitive, even for Euler flows. This notion has been and remains foreign to a large part of the research community in fluid dynamics. Furthermore, the pressure force responsible for driving local momentum has been known to be nonlinearly depleted to some extent [29]. It has been suggested that such depletion might be adequate to ensure non-singular growth of local momentum under viscous effects [29]. Hence, it is not excessively optimistic to hold firm to the belief that Navier-Stokes flows are regular and that the problem can be fruitfully handled by classical methods.

This study extends the results of Ref. [29] in several directions, by further examining the pressure force by both semi-analytic and fully analytic methods. Semi-analytic results, though inconclusive, are derived to illustrate that viscous effects may be strong enough to regularise flows evolving from sufficiently regular initial velocity fields. Owing to the fact that the pressure $p$ in the term that drives the dynamics of $\|u\|_{L^{q}}$, for $q \geq 3$, is determined up to some function of space and velocity, we introduce an effective pressure via such a function - a pressure moderator. This facilitates the search for optimal regularity criteria from the evolution equation for $\|u\|_{L^{q}}$, by varying the moderator within its admissible classes. Rigorous regularity criteria are derived with and without making use of the moderator. The results obtained in the absence of the moderator feature some improvement over existing criteria in the literature. Several criteria are derived in terms of the effective pressure and the results are interpreted. We discuss a simple moderation scheme and the plausibility of the present approach.

\section{BACKGROUND}

This section briefly reviews some classical results on which the present study is based. These include the Calderon-Zigmund inequality relating $u$ to $p$ and the Prodi-Serrin-Ladyzhenskaya regularity criterion in terms of $\|u\|_{L^{q}}$.

2.1. Basic estimates. By taking the divergence of the momentum equation in (1) and by virtue of $\nabla \cdot u=0$, we obtain

$$
\Delta p=-\nabla \cdot((u \cdot \nabla) u)=-\sum_{i, j=1}^{3} \partial_{i} \partial_{j}\left(u_{i} u_{j}\right) .
$$

Taking the gradient of (2) yields

$$
\Delta \nabla p=-\sum_{i, j=1}^{3} \partial_{i} \partial_{j}\left(\nabla\left(u_{i} u_{j}\right)\right) .
$$


These results allow us to use the Calderon-Zigmund inequality to estimate $p$ and $\nabla p$ in terms of $u$ and $\nabla u$ by

$$
\|p\|_{L^{q}} \leq c\|u\|_{L^{2 q}}^{2}, \text { for } 1<q<\infty
$$

and

$$
\|\nabla p\|_{L^{q}} \leq c\||u| \nabla u\|_{L^{q}}, \text { for } 1<q<\infty,
$$

where $c$ is a constant. Throughout this study, constants are denoted by $c$, which may assume different values from one expression to another.

By Hölder's inequality and (4) we have

$$
\left\|p|u|^{(q-2) / 2}\right\|_{L^{2}}^{2} \leq c\|u\|_{L^{q+2}}^{q+2} .
$$

By Sobolev's inequality we have (also see Ref. [29])

$$
\|u\|_{L^{3 q}}^{q / 2} \leq c q\left\||u|^{(q-2) / 2} \nabla|u|\right\|_{L^{2}} .
$$

We will use (6) and (7) for $q \geq 3$ in this study.

The evolution of the local energy $|u|^{2} / 2$ is governed by

$$
\frac{\partial}{\partial t} \frac{|u|^{2}}{2}+u \cdot \nabla \frac{|u|^{2}}{2}+u \cdot \nabla p=\Delta \frac{|u|^{2}}{2}-|\nabla u|^{2} .
$$

Integrating (8) over the domain yields the equation governing the decay of the global energy $\|u\|_{L^{2}}^{2} / 2$ :

$$
\frac{1}{2} \frac{\mathrm{d}}{\mathrm{d} t}\|u\|_{L^{2}}^{2}=-\|\nabla u\|_{L^{2}}^{2}=-\|\omega\|_{L^{2}}^{2}
$$

where the integrals of the advection, pressure and Laplacian terms identically vanish. Integrating (9) over time yields the bound

$$
\int_{0}^{t}\|\omega\|_{L^{2}}^{2} \mathrm{~d} \tau \leq \frac{\left\|u_{0}\right\|_{L^{2}}^{2}}{2}
$$

The quantity on the left-hand side of (10) represents cumulative energy dissipation and is among a few quantities that are a priori controlled. As far as the problem of regularity is concerned, equation (10) is a basis for estimates of relevant dynamical quantities.

2.2. The Prodi-Serrin-Ladyzhenskaya criterion. This subsection presents a derivation of the classical criterion $\int_{0}^{t}\|u\|_{L^{q}}^{2 q /(q-3)} d \tau<\infty$, for $q>3$. The steps leading to this result are also used in the subsequent calculations.

Multiplying the momentum equation in (1) by $-\Delta u$ and integrating the resulting equation over the domain yield

$$
\begin{aligned}
\frac{1}{2} \frac{\mathrm{d}}{\mathrm{d} t}\|\omega\|_{L^{2}}^{2} & \leq \int_{\mathbb{R}^{3}}|\Delta u\|u\| \nabla u| \mathrm{d} x-\|\Delta u\|_{L^{2}}^{2} \\
& \leq\|\Delta u\|_{L^{2}}\|u\|_{L^{q}}\|\nabla u\|_{L^{q^{\prime}}}-\|\Delta u\|_{L^{2}}^{2}
\end{aligned}
$$

where $1 / q+1 / q^{\prime}=1 / 2$. By using the Gagliardo-Nirenberg's inequality (a combination of Hölder's and Sobolev's inequalities in the present case), we can estimate the norm $\|\nabla u\|_{L^{q^{\prime}}}$ by

$$
\|\nabla u\|_{L^{q^{\prime}}} \leq c\|\nabla u\|_{L^{2}}^{1-3 / q}\|\Delta u\|_{L^{2}}^{3 / q} .
$$


Substituting this result into (11) yields

$$
\begin{aligned}
\frac{1}{2} \frac{\mathrm{d}}{\mathrm{d} t}\|\omega\|_{L^{2}}^{2} & \leq c\|\Delta u\|_{L^{2}}^{1+3 / q}\|\omega\|_{L^{2}}^{1-3 / q}\|u\|_{L^{q}}-\|\Delta u\|_{L^{2}}^{2} \\
& \leq c\|u\|_{L^{q}}^{2 q /(q-3)}\|\omega\|_{L^{2}}^{2}-c^{\prime}\|\Delta u\|_{L^{2}}^{2}
\end{aligned}
$$

where Young's inequality (again with $q>3$ ) has been used and $c^{\prime}$ is another constant. By applying Gronwall's lemma to (13) we obtain

$$
\|\omega\|_{L^{2}}^{2}+c^{\prime} \int_{0}^{t}\|\Delta u\|_{L^{2}}^{2} \mathrm{~d} \tau \leq\left\|\omega_{0}\right\|_{L^{2}}^{2} \exp \left\{c \int_{0}^{t}\|u\|_{L^{q}}^{2 q /(q-3)} \mathrm{d} \tau\right\} .
$$

It follows that $\|\omega\|_{L^{2}}^{2}$ is finite and $\|\Delta u\|_{L^{2}}^{2}$ is integrable provided that

$$
\int_{0}^{t}\|u\|_{L^{q}}^{2 q /(q-3)} \mathrm{d} \tau<\infty
$$

This condition turns out to be sufficient to prove finiteness of arbitrarily high order Sobolev norms of $u$, thereby ensuring regularity. The extension of (15) to the criterion $\operatorname{esssup}_{(0, \mathrm{t})}\|u\|_{L^{3}}<\infty$ requires a different approach [14], which is beyond the scope of the present study.

\section{Preliminary Results}

3.1. Viscous effects versus pressure force. From a physical point of view, viscous effects can be expected to adequately suppress growth of local momentum driven by the pressure gradient, at least for sufficiently regular velocity fields. The reasons can be seen from the latter of following two observations.

First, equation (8) implies that a fluid particle possessing a local maximum velocity can be accelerated only if $u \cdot \nabla p<0$, i.e. only if the particle moves toward lower pressure. Apparently, a blow-up of the fluid velocity would require infinite acceleration, i.e. $\widehat{u} \cdot \nabla p \rightarrow-\infty$, where $\widehat{u}=u /|u|$. Now since regularity persists whenever $\|p\|_{L^{\infty}}<\infty$, the only possibility for $\widehat{u} \cdot \nabla p \rightarrow-\infty$ is $p \rightarrow-\infty$. This result has been deduced by Seregin and Sverák on the basis of a more formal analysis [25].

Second, suppose $u \in L^{q}$, where $q>3$, so that the scaling of peak velocity within a ball of radius $r$ does not exceed $r^{-3 / q}$ in the limit $r \rightarrow 0$. As suggested by (4) and (5), given the scaling $r^{-3 / q}$ for $|u|$, the "forcing" term $\nabla p$ scales as $r^{-6 / q-1}$. Meanwhile, the viscosity term $\Delta u$ scales as $r^{-3 / q-2}$. For $q>3$, the latter apparently predominates the former. This means that whenever $u \in L^{q}$, where $q>3$, viscous effects are stronger than the pressure gradient force at peak velocity. As a consequence, $u$ remains in $L^{q}$ and regularity is assured. The critical case $q=3$ features an exact balance between the two forces and the present argument is inconclusive. This case corresponds to the well-known criterion $\operatorname{esssup}_{(0, \mathrm{t})}\|u\|_{L^{3}}<\infty$.

We now provide a more formal account of the above observation. Suppose that $|u|$ peaks (globally) at $x_{0}$. Let $B\left(x_{0}, r\right)$ be a ball centred at $x_{0}$ with radius $r$, which can be made as small as necessary. Figure 1 schematically depicts the flows in the vicinity of $x_{0}$. Integrating (8) over 


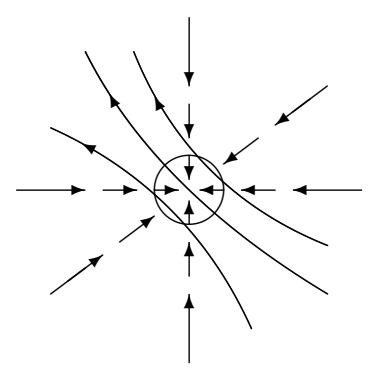

FiguRE 1. A schematic description of the flows in a neighbourhood of $x_{0}$ (located at the centre of the figure), where $|u|$ peaks. The circle represents a ball centred at $x_{0}$ with radius $r$. The arrows represent $\nabla|u|$, which vanishes at $x_{0}$, while the open curves represent streamlines.

$B\left(x_{0}, r\right)$ yields

$$
\begin{aligned}
\frac{1}{2} \frac{\mathrm{d}}{\mathrm{d} t} \int_{B}|u|^{2} \mathrm{~d} x & =-\int_{B} \nabla \cdot\left(\frac{|u|^{2}}{2} u+p u\right) \mathrm{d} x+\int_{B}\left(\Delta \frac{|u|^{2}}{2}-|\nabla u|^{2}\right) \mathrm{d} x \\
& =-\int_{\partial B}\left(\frac{|u|^{2}}{2} u+p u\right) \cdot \mathrm{d} s+\int_{\partial B} \nabla \frac{|u|^{2}}{2} \cdot \mathrm{d} s-\int_{B}|\nabla u|^{2} \mathrm{~d} x \\
& \leq-\int_{\partial B}\left(\frac{|u|^{2}}{2} u+p u\right) \cdot \mathrm{d} s-\int_{B}|\nabla u|^{2} \mathrm{~d} x,
\end{aligned}
$$

where $\partial B$ denotes the spherical boundary of $B$. In (16), the divergence theorem has been used and the second surface integral (expected to be non-positive for some small enough $r$ ) in the middle line has been omitted. Given $u \in L^{q}$, we have the scalings $\left|u\left(x_{0}\right)\right| \sim r^{-3 / q},|p| \sim\left|u\left(x_{0}\right)\right|^{2} \sim r^{-6 / q}$ and $|\nabla u| \sim r^{-3 / q-1}$. The first and second terms on the right-hand side of the final line scale as $r^{-9 / q+2}$ and $r^{-6 / q+1}$, respectively. In the limit $r \rightarrow 0$, the latter clearly predominates the former, provided that $q>3$. Hence, $\left|u\left(x_{0}\right)\right|$ decays and $u$ remains in $L^{q}$. Thus, there can be no loss of regularity.

Given a smooth $u_{0}(x)$, a divergence of $\left|u\left(x_{0}\right)\right| \sim r^{-3 / q}$ by $r \rightarrow 0$ without being accompanied by $q \rightarrow 3^{+}$is ruled out by the regularity criterion $\operatorname{esssup}_{(0, \mathrm{t})}\|u\|_{L^{3}}<\infty$. Hence a development of singularities would necessarily require $\left|u\left(x_{0}\right)\right|$ to (strongly) grow via both limits $r \rightarrow 0$ and $q \rightarrow 3^{+}$ in a concurrent fashion. The above arguments are applicable to this growth scenario. The upshot is that for smooth $u_{0}(x)$, highly concentrated momentum in the form $|u| \sim r^{-\epsilon}$, even for small $\epsilon>0$, may not develop in the first place.

Remark 1. If $\left|u\left(x_{0}\right)\right| \sim r^{-3 / q}$, for $q<3$, i.e. $u_{0}(x) \notin L^{3}$, then the pressure term predominates the viscosity term. The above arguments may not rule out the possibility $\left|u\left(x_{0}\right)\right| \rightarrow \infty$. This can be seen as an explanation for the critical nature of the criterion $\operatorname{essup}_{(0, \mathrm{t})}\|u\|_{L^{3}}<\infty$. As we do not expect rigorous analysis to "beat" the present semi-analytic arguments, this criterion may not be improved by standard energy method and analytic inequalities. However, the pressure force is known to have severe depletion of nonlinearity [29]. Hence, it seems plausible that the theory of Navier-Stokes regularity can progress within the current framework if such depletion can be exploited. 
Remark 2. Nonlinear depletion of the pressure force manifests itself through cancellation within the surface integral of pu in (16). Indeed, for sufficiently small $r, p$ may be assumed to have the same sign on $\partial B$. It then follows that inflow (flow into B) and outflow make contributions of opposite signs to the integral, hence cancelling each other to some extent.

3.2. Basic criteria. This subsection considers the evolution of $\|u\|_{L^{3}},\|u\|_{L^{4}}$ and $\|\omega\|_{L^{2}}$, from which two regularity criteria are derived. The results feature some improvement over existing ones.

For $q \geq 3$, the evolution of $\|u\|_{L^{q}}$ is governed by

$$
\begin{aligned}
\|u\|_{L^{q}}^{q-1} \frac{\mathrm{d}}{\mathrm{d} t}\|u\|_{L^{q}} & =-\int_{\mathbb{R}^{3}}|u|^{q-2} u \cdot \nabla p \mathrm{~d} x+\int_{\mathbb{R}^{3}}|u|^{q-2}\left(\Delta \frac{|u|^{2}}{2}-|\nabla u|^{2}\right) \mathrm{d} x \\
& =(q-2) \int_{\mathbb{R}^{3}} p|u|^{q-2} \widehat{u} \cdot \nabla|u| \mathrm{d} x-(q-2)\left\||u|^{(q-2) / 2} \nabla|u|\right\|_{L^{2}}^{2}-\left\||u|^{(q-2) / 2} \nabla u\right\|_{L^{2}}^{2} .
\end{aligned}
$$

When $q=3$, equation (17) reduces to

$$
\frac{\mathrm{d}}{\mathrm{d} t}\|u\|_{L^{3}} \leq \frac{1}{\|u\|_{L^{3}}^{2}} \int_{\mathbb{R}^{3}}|p\|u\| \nabla| u\left\|\mathrm{~d} x \leq \frac{c\|u\|_{L^{6}}^{3}\|\omega\|_{L^{2}}}{\|u\|_{L^{3}}^{2}} \leq \frac{c\|\omega\|_{L^{2}}^{4}}{\|u\|_{L^{3}}^{3}}\right\| u \|_{L^{3}},
$$

where Hölder's inequality, (6) and Sobolev's inequality have been used. Integrating (18) over time yields the following criterion.

Theorem 1. Let $u$ and $p$ solve the Navier-Stokes equations (1). If

$$
\int_{0}^{t} \frac{\|\omega\|_{L^{2}}^{4}}{\|u\|_{L^{3}}^{3}} \mathrm{~d} \tau<\infty
$$

then

$$
\|u\|_{L^{q}} \leq\left\|u_{0}\right\|_{L^{q}} \exp \left\{c \int_{0}^{t} \frac{\|\omega\|_{L^{2}}^{4}}{\|u\|_{L^{3}}^{3}} \mathrm{~d} \tau\right\}<\infty
$$

and regularity follows.

This slightly improves a previous result of Ref. [29] by a factor of $\|u\|_{L^{3}}^{-1}$. Interestingly, the improvement is possible by not using the dissipation terms in the evolution equation for $\|u\|_{L^{3}}$.

It is beneficial to consider a combination of the evolution equations for $\|u\|_{L^{4}}$ and $\|\omega\|_{L^{2}}$, for the reason to become obvious in due course. For $q=4$, equation (17) becomes

$$
\begin{aligned}
\|u\|_{L^{4}}^{3} \frac{\mathrm{d}}{\mathrm{d} t}\|u\|_{L^{4}} & =2 \int_{\mathbb{R}^{3}} p|u|^{2} \widehat{u} \cdot \nabla|u| \mathrm{d} x-2\||u| \nabla|u|\|_{L^{2}}^{2}-\||u| \nabla u\|_{L^{2}}^{2} \\
& \leq 2\|p u\|_{L^{2}}\|u \cdot \nabla|u|\|_{L^{2}}-2\||u| \nabla|u|\|_{L^{2}}^{2}-\||u| \nabla u\|_{L^{2}}^{2} \\
& \leq \frac{1}{2}\|p u\|_{L^{2}}^{2}-\||u| \nabla u\|_{L^{2}}^{2} .
\end{aligned}
$$

Meanwhile, equation (11) can be rewritten in the form

$$
\begin{aligned}
\frac{1}{2} \frac{\mathrm{d}}{\mathrm{d} t}\|\omega\|_{L^{2}}^{2} & \leq\|\Delta u\|_{L^{2}}\|(u \cdot \nabla) u\|_{L^{2}}-\|\Delta u\|_{L^{2}}^{2} \\
& \leq\|\Delta u\|_{L^{2}}\|u \mid \nabla u\|_{L^{2}}-\|\Delta u\|_{L^{2}}^{2} \\
& \leq\||u| \nabla u\|_{L^{2}}^{2}-\frac{3}{4}\|\Delta u\|_{L^{2}}^{2} .
\end{aligned}
$$


Adding (21) and (22) yields

$$
\begin{aligned}
\frac{\mathrm{d}}{\mathrm{d} t}\|u\|_{L^{4}}^{4}+2 \frac{\mathrm{d}}{\mathrm{d} t}\|\omega\|_{L^{2}}^{2} & \leq 2\|p u\|_{L^{2}}^{2}-3\|\Delta u\|_{L^{2}}^{2} \leq c\|u\|_{L^{6}}^{6}-3\|\Delta u\|_{L^{2}}^{2} \\
& =\frac{c\|u\|_{L^{6}}^{6}}{\|u\|_{L^{4}}^{4}+2\|\omega\|_{L^{2}}^{2}}\left(\|u\|_{L^{4}}^{4}+2\|\omega\|_{L^{2}}^{2}\right)-3\|\Delta u\|_{L^{2}}^{2},
\end{aligned}
$$

where equation (6) has been used. From this estimate, we have the following theorems.

Theorem 2. Let $u$ and $p$ solve the Navier-Stokes equations (1). If

$$
\int_{0}^{t} \frac{\|u\|_{L^{6}}^{6}}{\|u\|_{L^{4}}^{4}+2\|\omega\|_{L^{2}}^{2}} \mathrm{~d} \tau<\infty
$$

then both $\|u\|_{L^{4}}$ and $\|\omega\|_{L^{2}}$ remain finite and regularity follows.

Theorem 3. Let $u$ and $p$ solve the Navier-Stokes equations (1). There exists $c>0$ such that if

$$
\|u\|_{L^{6}} \leq c\|\Delta u\|_{L^{2}}^{1 / 3},
$$

then either $\|u\|_{L^{4}}$ or $\|\omega\|_{L^{2}}$ (or both) decays and regularity follows.

Remark 3. While both theorems 2 and 3 make use of the dissipation term $\|\Delta u\|_{L^{2}}^{2}$, the latter exploits this term more fully than the former.

Remark 4. By setting $q=6$ and $q^{\prime}=3$ in (11) and after some simple manipulation, we arrive at the conclusion that $\|\omega\|_{L^{2}}$ decays when $\|u\|_{L^{6}} \leq c\|\Delta u\|_{L^{2}}^{1 / 4}$. This means that criterion (25) represents a "gain" in optimality by a factor of $\|\Delta u\|_{L^{2}}^{1 / 12}$. This gain is due to the fact that in (22), the vortex stretching term has been estimated as a quadratic rather than a cubic.

Remark 5. Criterion (25) may be replaced by the following version

$$
\int_{0}^{t}\|u\|_{L^{6}}^{6} \mathrm{~d} \tau \leq c \int_{0}^{t}\|\Delta u\|_{L^{2}}^{2} \mathrm{~d} \tau .
$$

Remark 6. Criterion (25) remains unchanged under the scalings $p(x, t) \longmapsto \lambda^{2} p\left(\lambda x, \lambda^{2} t\right)$ and $u(x, t) \longmapsto \lambda u\left(\lambda x, \lambda^{2} t\right)$ that render (1) invariant. Interestingly, the quantities on the left- and righthand sides of (25) are, respectively, of large $(u)$ and very small scales $(\Delta u)$, in comparison with the small scales represented by $\omega$.

Consider the following version of the Gagliardo-Nirenberg inequality

$$
\|u\|_{L^{6}} \leq c\|\Delta u\|_{L^{2}}^{(6-q) /(6+q)}\|u\|_{L^{q}}^{2 q /(6+q)},
$$

which is valid for $1 \leq q \leq 6$. In what follows, equation (27) is used with $q \in(3,6]$. We have

$$
\begin{aligned}
\int_{0}^{t}\|u\|_{L^{6}}^{6} \mathrm{~d} \tau & \leq c \int_{0}^{t}\|\Delta u\|_{L^{2}}^{6(6-q) /(6+q)}\|u\|_{L^{q}}^{12 q /(6+q)} \mathrm{d} \tau \\
& \leq c\left(\int_{0}^{t}\|\Delta u\|_{L^{2}}^{2} \mathrm{~d} \tau\right)^{3(6-q) /(6+q)}\left(\int_{0}^{t}\|u\|_{L^{q}}^{3 q /(q-3)} \mathrm{d} \tau\right)^{4(q-3) /(6+q)},
\end{aligned}
$$

where Hölder's inequality has been used. It follows that equation (26) holds if

$$
\int_{0}^{t}\|u\|_{L^{q}}^{3 q /(q-3)} \mathrm{d} \tau \leq c \int_{0}^{t}\|\Delta u\|_{L^{2}}^{2} \mathrm{~d} \tau .
$$

Hence we have the following corollary. 
Corollary 1. Let $u$ and $p$ solve the Navier-Stokes equations (1). If

$$
\int_{0}^{t}\|u\|_{L^{q}}^{3 q /(q-3)} \mathrm{d} \tau \leq c \int_{0}^{t}\|\Delta u\|_{L^{2}}^{2} \mathrm{~d} \tau,
$$

then either $\|u\|_{L^{4}}$ or $\|\omega\|_{L^{2}}$ (or both) decays and regularity follows.

\section{Pressure moderation and main Results}

The pressure $p$ in (1) is determined up to an arbitrary function of $t$. This uninteresting feature is removed by the condition of decay at infinity. What is remarkable is that in (17), $p$ is determined up to a function of $x$ and $|u|$, say $\mathcal{P}(x,|u|)$, which can assume relatively broad forms. As far as the problem of optimal bounds for the pressure term in (17) are concerned, $\mathcal{P}(x,|u|)$ presents itself as a powerful tool. The present study considers using $\mathcal{P}(x,|u|)$ to moderate $p$ in the vicinity of peak velocity, where $|p|$ is also expected to peak. Several regularity criteria are derived in terms of the "effective" (moderated) pressure $p+\mathcal{P}$.

We first derive a simple identity concerning the form of $\mathcal{P}$. The result is summarised in the following lemma.

Lemma 1. Let

$$
\mathcal{P}(x,|u|)=f(x) g(|u|),
$$

where

$$
u \cdot \nabla f(x)=0 \text { and } g(|u|) \in C^{1},
$$

then

$$
\int_{\mathbb{R}^{3}} \mathcal{P}|u|^{q-2} \widehat{u} \cdot \nabla|u| \mathrm{d} x=0 .
$$

Proof. Let

$$
G(s)=\frac{1}{s^{q-2}} \int_{0}^{s} \xi^{q-3} g(\xi) \mathrm{d} \xi .
$$

By virtue of $\nabla \cdot u=0$ and the hypothesis $u \cdot \nabla f(x)=0$, we have

$$
\begin{aligned}
\nabla \cdot\left(f(x) G(|u|)|u|^{q-2} u\right) & =f(x) u \cdot \nabla \int_{0}^{|u|} \xi^{q-3} g(\xi) \mathrm{d} \xi \\
& =f(x) g(|u|)|u|^{q-2} \widehat{u} \cdot \nabla|u| .
\end{aligned}
$$

Integrating (32) over $\mathbb{R}^{3}$ proves the lemma.

Remark 7. Lemma 1 applies to a bounded domain $D$ with nonslip boundary $\partial D \in C^{1}$.

Recall (17)

$$
\begin{aligned}
\|u\|_{L^{q}}^{q-1} \frac{\mathrm{d}}{\mathrm{d} t}\|u\|_{L^{q}}= & (q-2) \int_{\mathbb{R}^{3}}(p+\mathcal{P})|u|^{q-2} \widehat{u} \cdot \nabla|u| \mathrm{d} x \\
& -(q-2)\left\||u|^{(q-2) / 2} \nabla|u|\right\|_{L^{2}}^{2}-\left\||u|^{(q-2) / 2} \nabla u\right\|_{L^{2}}^{2},
\end{aligned}
$$

where the vanishing term (31) has been inserted. The introduction of this term enables us to seek optimal estimates for the pressure term by varying $\mathcal{P}$, i.e. varying $f(x) g(|u|)$ within its admissible classes. For this reason, $\mathcal{P}$ and $p+\mathcal{P}$ are called the pressure moderator and effective pressure, respectively. There are essentially two admissible classes for $f(x) g(|u|): g(|u|)$ and $f(x) g(|u|)$, where the former is obtained by setting $f(x)=1$. The hypothesis $u \cdot \nabla f(x)=0$ requires $f(x)$ to be constant 
along streamlines. This appears to be a stiff constraint on $f(x)$, which, nonetheless represents a "degree of freedom" of the moderator. On the other hand, another degree of freedom of the moderator is provided by $g(|u|)$, which can be flexible enough for the intended purpose as it is subject to virtually no conditions, except for decay at infinity as $|u|$ itself. As the very term "moderator" suggests, we are mainly interested in $\mathcal{P}$ such that $|p+\mathcal{P}| \leq|p|$ in $\Omega$ (ideally $|p+\mathcal{P}| \ll|p|$ ). Hence we may assume in some subsequent calculations that $\|p+\mathcal{P}\|_{L^{q}} \approx\|p\|_{L^{q}} \leq c\|u\|_{L^{2 q}}^{2}$. Note that linear combinations of $|u|^{\alpha}$, for $0 \leq \alpha \leq 2$, are admissible forms for $g(|u|)$. Of particular interest is $g(|u|)=|u|^{2}$, so that $f(x) g(|u|)=f(x)|u|^{2}$, which may partially neutralise $p$ in the vicinity of its minimum (negative) since $|u|^{2}$ is expected to be comparable to $|p|$ in such vicinity.

It has been widely recognised in the turbulence community that rotation (in conjunction with density stratification) has "regularising effects" on fluid turbulence, by tending to suppress the turbulence in the direction of rotation (and stratification). This phenomenon has been known as two-dimensionalisation. In the present context, regularising effects of rotation may be readily appreciated by the fact that fluid particles are deflected away from the paths of optimal acceleration under the sideways influence of the Coriolis force. Such a force is given by $|u| A \widehat{u}$, where $A$ is an anti-symmetric matrix. Now consider the force term $|u|^{2} \nabla f(x)$, which, when added to (1), has similar effects as $|u| A \widehat{u}$ (in the sense that both are perpendicular to $u$ ), albeit relatively stronger due to its quadratic rather than linear dependence on $|u|$. The moderator $f(x)|u|^{2}$ can be seen to be attributable to the force term $|u|^{2} \nabla f(x)$. This gives some insight into the pressure term and the present notion of pressure moderation.

In order to fully exploit $\mathcal{P}$, we divide space into regions of high and low velocity. Similar to Tran and $\mathrm{Yu}[29]$, we consider a partition of $\mathbb{R}^{3}$ in the following manner. For $q \geq 3$, let $\Omega \subset \mathbb{R}^{3}$ be such that the following conditions hold.

$$
\begin{aligned}
& |u|>\left(\frac{c}{q^{2}} \frac{\|u\|_{L^{3 q-\gamma}}^{q / \beta}}{\|p+\mathcal{P}\|_{L^{2}}^{2}\|u\|_{L^{2}}^{q \alpha / \beta}}\right)^{1 /(q-2)}, \text { for } x \in \Omega ; \\
& \text { - }|u| \leq\left(\frac{c}{q^{2}} \frac{\|u\|_{L^{3 q-\gamma}}^{q / \beta}}{\|p+\mathcal{P}\|_{L^{2}}^{2}\|u\|_{L^{2}}^{q \alpha / \beta}}\right)^{1 /(q-2)} \quad, \text { for } x \in \Omega^{c}=\mathbb{R}^{3} \backslash \Omega .
\end{aligned}
$$

Here $\gamma>0$ is a parameter (to be determined shortly) and $\alpha$ and $\beta$ are (two Hölder conjugate exponents appearing in a Hölder inequality below) given by

$$
\alpha=\frac{2 \gamma}{(3 q-\gamma)(3 q-2)} \text { and } \beta=\frac{3 q(3 q-\gamma-2)}{(3 q-\gamma)(3 q-2)} .
$$

Hereafter, the above partition is referred to as $\mathbf{P}$ for convenience. For $q=3$ we set $\gamma=5$. The bound for $|u|$ in $\mathbf{P}$ then becomes (constants omitted)

$$
\frac{\|u\|_{L^{4}}^{14 / 3}}{\|p+\mathcal{P}\|_{L^{2}}^{2}\|u\|_{L^{2}}^{5 / 3}} \geq \frac{\|u\|_{L^{4}}^{14 / 3}}{c\|u\|_{L^{4}}^{4}\|u\|_{L^{2}}^{5 / 3}}=\frac{\|u\|_{L^{4}}^{2 / 3}}{c\|u\|_{L^{2}}^{5 / 3}} \geq \frac{\|u\|_{L^{3}}}{c\|u\|_{L^{2}}^{2}} .
$$

Hence $\Omega$ consists of regions with velocity of order $\|u\|_{L^{3}}$. Meanwhile, for $q \geq 4$ we set $\gamma=6$. The bound for $|u|$ in $\mathbf{P}$ then becomes (again constants omitted)

$$
\left(\frac{\|u\|_{L^{3 q-6}}^{(q-2)(3 q-2) /(3 q-8)}}{\|p+\mathcal{P}\|_{L^{2}}^{2}\|u\|_{L^{2}}^{4 /(3 q-8)}}\right)^{1 /(q-2)} \geq\left(\frac{\|u\|_{L^{3 q-6}}^{(q-2)(3 q-2) /(3 q-8)}}{c\|u\|_{L^{4}}^{4}\|u\|_{L^{2}}^{4 /(3 q-8)}}\right)^{1 /(q-2)} \geq \frac{\|u\|_{L^{3 q-6}}}{\left(c\|u\|_{L^{2}}^{2}\right)^{1 /(q-2)}}
$$


This ensures that $\Omega$ consists of regions with velocity of order $\|u\|_{L^{3 q-6}}$. Note that in the limit $q \rightarrow \infty$, the bound in $\mathbf{P}$ tends to $\|u\|_{L^{\infty}}$.

Remark 8. The assumption $\|p+\mathcal{P}\|_{L^{2}} \leq c\|u\|_{L^{4}}^{2}$ is necessary for the case $q=3$ to ensure that $\Omega$ consists of regions with velocity in the order of $\|u\|_{L^{3}}$. When $q \geq 4$, this assumption means $\Omega$ consists of regions with velocity in the order of $\|u\|_{L^{3 q-6}}$. It follows that if we just require the velocity in $\Omega$ to be in the order of $\|u\|_{L^{q}}$, then the requirement on $\|p+\mathcal{P}\|_{L^{2}}$ can be a bit more relaxing.

It can be seen that $\mathbf{P}$ is designed in such a way that the contribution from the region $\Omega^{c}$ to the pressure term in (33) can be cancelled out by the dissipation terms. Hence the contribution from $\Omega$ is solely responsible for growth of $\|u\|_{L^{q}}$. Indeed, we have

$$
\begin{aligned}
\int_{\Omega^{c}}(p+\mathcal{P})|u|^{q-2} \widehat{u} \cdot \nabla|u| \mathrm{d} x & \leq\left(\int_{\Omega^{c}}(p+\mathcal{P})^{2}|u|^{q-2} \mathrm{~d} x\right)^{1 / 2}\left\||u|^{(q-2) / 2} \nabla|u|\right\|_{L^{2}} \\
& \leq \frac{c}{q} \frac{\|u\|_{L^{3 q-\gamma}}^{q /(2 \beta)}}{\|u\|_{L^{2}}^{q \alpha /(2 \beta)}}\left\||u|^{(q-2) / 2} \nabla|u|\right\|_{L^{2}} \\
& \leq \frac{c}{q}\|u\|_{L^{3 q}}^{q / 2}\left\||u|^{(q-2) / 2} \nabla|u|\right\|_{L^{2}} \\
& \leq c\left\||u|^{(q-2) / 2} \nabla|u|\right\|_{L^{2}}^{2}
\end{aligned}
$$

where the Hölder inequality

$$
\|u\|_{L^{3 q-\gamma}} \leq\|u\|_{L^{2}}^{\alpha}\|u\|_{L^{3 q}}^{\beta}
$$

and (7) have been used. Substituting (34) into (33) yields

$$
\begin{aligned}
\|u\|_{L^{q}}^{q-1} \frac{\mathrm{d}}{\mathrm{d} t}\|u\|_{L^{q}} \leq & (q-2) \int_{\Omega}(p+\mathcal{P})|u|^{q-2} \widehat{u} \cdot \nabla|u| \mathrm{d} x-c(q-2)\left\||u|^{(q-2) / 2} \nabla \mid u\right\|_{L^{2}}^{2} \\
& -\left\||u|^{(q-2) / 2} \nabla u\right\|_{L^{2}}^{2} .
\end{aligned}
$$

The integral over $\Omega$ (by itself or in conjunction with the dissipation terms) is susceptible of several different estimates, the relative optimality of which is not known a priori. In what follows, we consider two estimates, each with a reasonable "weight" on the effective pressure, banking on the possibility that this pressure can be made moderate in $\Omega$.

The case $q=3$ admits a special estimate, which deserves separate attention. Otherwise, we handle all cases $q \geq 3$ together. For $q=3$, equation (35) becomes

$$
\begin{aligned}
\|u\|_{L^{3}}^{2} \frac{\mathrm{d}}{\mathrm{d} t}\|u\|_{L^{3}} \leq & \int_{\Omega}(p+\mathcal{P})|u| \widehat{u} \cdot \nabla|u| \mathrm{d} x-c\left\||u|^{1 / 2} \nabla|u|\right\|_{L^{2}}^{2}-\left\||u|^{1 / 2} \nabla u\right\|_{L^{2}}^{2} \\
\leq & \left(\int_{\Omega}(p+\mathcal{P})^{4} \mathrm{~d} x\right)^{1 / 4}\|u\|_{L^{2}}^{1 / 2}\left\||u|^{1 / 2} \nabla|u|\right\|_{L^{2}} \\
& -c\left\||u|^{1 / 2} \nabla|u|\right\|_{L^{2}}^{2}-\left\||u|^{1 / 2} \nabla u\right\|_{L^{2}}^{2},
\end{aligned}
$$

where Hölder's inequality has been used. If the right-hand side of (36) is negative then $\|u\|_{L^{3}}$ decays and we have the following theorem. 
Theorem 4. Let $u$ and $p$ solve the Navier-Stokes equations (1). If

$$
\int_{\Omega}(p+\mathcal{P})^{4} \mathrm{~d} x \leq c\left\||u|^{1 / 2} \nabla u\right\|_{L^{2}}^{4},
$$

where $\Omega$ is defined by partition $\mathbf{P}$ and $\mathcal{P}$ is described by lemma 1 , then $\|u\|_{L^{3}}$ decays and regularity follows.

Remark 9. The criterion in theorem 4 can be stated in the form

$$
\int_{0}^{t}\left(\int_{\Omega}(p+\mathcal{P})^{4} \mathrm{~d} x\right)^{1 / 2} \mathrm{~d} \tau \leq c \int_{0}^{t}\left\||u|^{1 / 2} \nabla u\right\|_{L^{2}}^{2} \mathrm{~d} \tau .
$$

We now consider $q \geq 3$, with an emphasis on the limit of large $q$. This limit is appealing since $\Omega$ reduces in size, to a point if $|u|$ peaks at a single point. Intuitively, $p$ will be easier to moderate in this limit. The price is the magnitude of $q$, which appears in the results. This turns out to be an issue unresolved in the present study. A similar issue involving the limit $q \rightarrow \infty$ is discussed in some detail in Ref. [29].

From (35) we have

$$
\begin{aligned}
\|u\|_{L^{q}}^{q-1} \frac{\mathrm{d}}{\mathrm{d} t}\|u\|_{L^{q}} \leq & (q-2)\left(\int_{\Omega} \frac{|p+\mathcal{P}|^{3}}{|u|^{3}} \mathrm{~d} x\right)^{1 / 3}\|u\|_{L^{3 q}}^{q / 2}\left\||u|^{(q-2) / 2} \nabla u\right\|_{L^{2}} \\
& -c(q-2)\left\||u|^{(q-2) / 2} \nabla|u|\right\|_{L^{2}}^{2}-\left\||u|^{(q-2) / 2} \nabla u\right\|_{L^{2}}^{2} \\
\leq & c^{\prime} q(q-2)\left(\int_{\Omega} \frac{|p+\mathcal{P}|^{3}}{|u|^{3}} \mathrm{~d} x\right)^{1 / 3}\left\||u|^{(q-2) / 2} \nabla u\right\|_{L^{2}}^{2} \\
& -c(q-2)\left\||u|^{(q-2) / 2} \nabla|u|\right\|_{L^{2}}^{2}-\left\||u|^{(q-2) / 2} \nabla u\right\|_{L^{2}}^{2},
\end{aligned}
$$

where Hölder's inequality and (7) have been used. This result immediately implies the following theorem.

Theorem 5. Let $u$ and $p$ solve the Navier-Stokes equations (1). There is $c>0$ such that if

$$
\int_{\Omega} \frac{|q(p+\mathcal{P})|^{3}}{|u|^{3}} \mathrm{~d} x \leq c,
$$

where $\Omega$ is defined by partition $\mathbf{P}$ and $\mathcal{P}$ is given by lemma 1 , then $\|u\|_{L^{q}}$ decays and regularity follows.

In the limit $q \rightarrow \infty, \Omega$ reduces to the point(s) of peak $|u|$. Hence, its measure, say $|\Omega|$, tends to zero. An obvious moderation scheme is such that $p \rightarrow-\mathcal{P}$, i.e. $p+\mathcal{P} \rightarrow 0$ toward peak $|u|$. Regularity requires this approach and $|\Omega| \rightarrow 0$ to be rapid enough, so that the integral in (39) tends to zero. Now, consider a finite $q \geq 3$. In the limit $\|u\|_{L^{q}} \rightarrow \infty$, we also have $|\Omega| \rightarrow 0$. It follows that if $|p+\mathcal{P}| \approx|u|^{5 / 3}$ in $\Omega$, then

$$
\int_{\Omega} \frac{|p+\mathcal{P}|^{3}}{|u|^{3}} \mathrm{~d} x \approx \int_{\Omega}|u|^{2} \mathrm{~d} x \rightarrow 0 .
$$

Therefore, equation (39) holds and regularity is secured. In other words, a moderation scheme that brings $|p|$ from, presumably, $|u|^{2}$ down to $|u|^{5 / 3}$ is required. It is possible to reformulate theorem 5 in such a way that criterion (39) becomes a Prodi-Serrin type. This has been presented in [30], where we essentially obtain a localized condition for $\operatorname{esssup}_{(0, t)}\|p\|_{L^{3 / 2}}$. 
The preceding result has made use of estimates that decouple all factors in the pressure term. In what follows, we keep $p+\mathcal{P}$ and $\widehat{u} \cdot \nabla|u|$ together, for the reason to become obvious shortly. Thus, instead of (38) we have

$$
\begin{aligned}
\|u\|_{L^{q}}^{q-1} \frac{\mathrm{d}}{\mathrm{d} t}\|u\|_{L^{q}} \leq & (q-2) \int_{\Omega} \frac{p+\mathcal{P}}{|u|^{2}}|u|^{q} \widehat{u} \cdot \nabla|u| \mathrm{d} x \\
& -c(q-2)\left\||u|^{(q-2) / 2} \nabla|u|\right\|_{L^{2}}^{2}-\left\||u|^{(q-2) / 2} \nabla u\right\|_{L^{2}}^{2} \\
\leq & (q-2)\left(\int_{\Omega}\left|\frac{p+\mathcal{P}}{|u|^{2}} \widehat{u} \cdot \nabla\right| u||^{3 / 2} \mathrm{~d} x\right)^{2 / 3}\|u\|_{L^{3 q}}^{q} \\
& -c(q-2)\left\|\left.|u|^{(q-2) / 2} \nabla|u|\right|_{L^{2}} ^{2}-\right\||u|^{(q-2) / 2} \nabla u \|_{L^{2}}^{2} \\
\leq & c^{\prime} q^{2}(q-2)\left(\int_{\Omega}\left|\frac{p+\mathcal{P}}{|u|^{2}} \widehat{u} \cdot \nabla\right| u||^{3 / 2} \mathrm{~d} x\right)^{2 / 3}\left\||u|^{(q-2) / 2} \nabla|u|\right\|_{L^{2}}^{2} \\
& -c(q-2)\left\||u|^{(q-2) / 2} \nabla|u|\right\|_{L^{2}}^{2}-\left\||u|^{(q-2) / 2} \nabla u\right\|_{L^{2}}^{2}
\end{aligned}
$$

where Hölder's inequality and (7) have been used. This result immediately implies the following theorem.

Theorem 6. Let $u$ and $p$ solve the Navier-Stokes equations (1). There is $c>0$ such that if

$$
\int_{\Omega}\left|q^{2} \frac{p+\mathcal{P}}{|u|^{2}} \widehat{u} \cdot \nabla\right| u||^{3 / 2} \mathrm{~d} x \leq c,
$$

where $\Omega$ is defined by partition $\mathbf{P}$ and $\mathcal{P}$ is given by lemma 1 , then $\|u\|_{L^{q}}$ decays and regularity follows.

In the limit $q \rightarrow \infty$, we have $\widehat{u} \cdot \nabla|u| \rightarrow 0$ and $|\Omega| \rightarrow 0$. As in the preceding case, we seek an admissible $\mathcal{P}$ such that $p+\mathcal{P} \rightarrow 0$ toward peak $|u|$. We then have both $p+\mathcal{P} \rightarrow 0$ and $\widehat{u} \cdot \nabla|u| \rightarrow 0$, together with $|\Omega| \rightarrow 0$, against $q \rightarrow \infty$. Now, consider a finite $q \geq 3$ and the limit $\|u\|_{L^{q}} \rightarrow \infty$. Suppose that there exists a moderation scheme that results in $|(p+\mathcal{P}) \widehat{u} \cdot \nabla| u|| \approx|u|^{10 / 3}$ in $\Omega$. Then

$$
\int_{\Omega}\left|\frac{p+\mathcal{P}}{|u|^{2}} \widehat{u} \cdot \nabla\right| u||^{3 / 2} \mathrm{~d} x \approx \int_{\Omega}|u|^{2} \mathrm{~d} x \rightarrow 0 .
$$

Therefore, equation (41) holds and regularity is secured. Note that in this case, the above scheme that $p+\mathcal{P}$ vanishes toward peak $|u|$ may still work, although the focus of the present moderation is "off peak" rather than "on peak" velocity.

Finally, we modify the calculations leading to theorems 2 and 3 with a pressure moderator. By combining (22) with (35), we obtain

$$
\begin{aligned}
\frac{\mathrm{d}}{\mathrm{d} t}\left(\|u\|_{L^{4}}^{4}+2\|\omega\|_{L^{2}}^{2}\right) & \leq 8 \int_{\Omega}(p+\mathcal{P})|u|^{2} \widehat{u} \cdot \nabla|u| \mathrm{d} x-8 c\||u| \nabla|u|\|^{2}-3\|\Delta u\|_{L^{2}}^{2} \\
& \leq c \int_{\Omega}(p+\mathcal{P})^{2}|u|^{2} \mathrm{~d} x-\|\Delta u\|_{L^{2}}^{2}
\end{aligned}
$$

where Young's inequality has been used. This immediately gives the following theorem 
Theorem 7. Let $u$ and $p$ solve the Navier-Stokes equations (1). If

$$
\int_{\Omega}(p+\mathcal{P})^{2}|u|^{2} \mathrm{~d} x \leq c\|\Delta u\|_{L^{2}}^{2},
$$

where $\Omega$ is defined by partition $\mathbf{P}$ and $\mathcal{P}$ is described by lemma 1, then either $\|u\|_{L^{4}}$ or $\|\omega\|_{L^{2}}$ (or both) decays and regularity follows.

Remark 10. The criterion in theorem 7 can be stated in the time-average sense.

We conclude this study with some remarks on a possible pressure moderation scheme. Note that an alternative notion of pressure moderation is that of pressure approximation in terms of a moderator $f g$. Intuitively, detailed knowledge of the relation between $p$ and $|u|$ within $\Omega$ is crucial for the approximation process. In general, available information on such relation is provided by (2), (3), (4) and (5), where equation (2) can also be expressed in the form

$$
\Delta\left(p+\frac{|u|^{2}}{2}\right)=\nabla \cdot(u \times \omega) .
$$

Ideally, if the Poission equation (44) can be solved and its solution can be approximated in terms of $f g$, then we have a moderator. In unfavourable circumstances, the following scheme merits some thoughts and further investigation.

For simplicity we consider the simple moderator $f(x)|u|^{2}$ and assume that $|u|$ peaks at a single point $x_{0}$. Consider a simple surface $S$ (a plane for example) passing through $x_{0}$. For each $x \in S \cap \Omega$, let $\ell_{x}$ be the portion of the streamline passing through $x$ and contained within $\Omega$. Now for $y \in \ell_{x}$, we define

$$
f(y)=-\frac{\int_{\ell_{x}} p(y) \mathrm{d} \ell}{\int_{\ell_{x}}|u(y)|^{2} \mathrm{~d} \ell} .
$$

It follows that

$$
p(x)+f(x)|u(x)|^{2}=0,
$$

for $x$ on a surface in $\Omega$. Note that since $f(x)$ is constant on $\ell_{x}$, we have $u \cdot \nabla f(x)=0$ as required. This makes $\mathcal{P}=f(x)|u|^{2}$ a plausible moderator.

\section{Concluding Remarks}

For Navier-Stokes flows evolving from sufficiently regular initial velocity fields, the criterion $\|u\|_{L^{3}}<\infty$ unambiguously implies that no loss of regularity occurs if $u$ remains in $L^{3}$. This study aims to understand the question of whether or not the pressure force is capable of "driving" a sufficiently regular velocity field $u$ out of $L^{3}$. An almost negative answer has been deduced on the basis of semi-analytic arguments. An effective pressure has been introduced via an additive moderator to replace the genuine pressure in the term that drives the dynamics of $\|u\|_{L^{q}}$, for $q \geq 3$. This approach allows one to vary the moderator within its admissible classes in the search for optimal bounds for that driving term. New regularity criteria have been derived by standard energy method with and without pressure moderation. In the absence of the moderator, some slight improvement on existing results has been achieved by considering the evolution of the hybrid quantity $\|u\|_{L^{4}}^{4}+2\|\omega\|_{L^{2}}^{2}$. This essentially allows us to estimate the vortex stretching term in the evolution equation for $\|\omega\|_{L^{2}}$ as a quadratic rather than a cubic, thereby making the improvement possible. Several new criteria have been derived in terms of the effective pressure. A simple 
moderator has been considered. The results appear promising, pending theoretical establishment of an effective moderation scheme.

Acknowledgments. This paper was presented at the 3rd Scottish PDE Colloquium (Strathclyde) under the title "A physicist's approach to Navier-Stokes regularity."

\section{REFERENCES}

[1] Beirao da Veiga H and Berselli L C 2002 On the regularizing effect of the vorticity direction in incompressible viscous flows Diff. Integral Eqns 15 345-356

[2] Berselli L C 2009 Some geometric constraints and the problem of global regularity for the Navier-Stokes equations Nonlinearity 22 2561-2581

[3] Berselli L C and Galdi G P 2002 Regularity criteria involving the pressure for the weak solutions to the Navier-Stokes equations Proc. Amer. Math. Soc. 130 3585-3595

[4] Caffarelli L, Kohn R and Nirenberg L 1982 Partial regularity of suitable weak solutions of the Navier-Stokes equations Commun. Pure Appl. Math. 35 771-831

[5] CAO C 2010 Sufficient conditions for the regularity to the $3 D$ Navier-Stokes equations Discrete Contin. Dyn. Syst. 26 1141-1151

[6] CaO C and Titi E S 2008 Regularity criteria for the three-dimensional Navier-Stokes equations Indiana Univ. Math. J. 57 2663-2680

[7] CaO C and Titi E S 2011 Global regularity criterion for the $3 D$ Navier-Stokes equations involving one entry of the velocity gradient tensor Arch. Ration. Mech. Anal. 202 919-932

[8] Chae D and Lee J 2001 Regularity in terms of pressure for the Navier-Stokes equations Nonlinear Anal. 46 $727-735$

[9] Chan C H And Yoneda T 2012 On possible isolated blow-up phenomena and regularity criterion of the 3D Navier-Stokes equation along the streamlines Methods Appl. Anal. 19 211-242

[10] Constantin P and Fefferman C 1993 Direction of vorticity and the problem of global regularity for the Navier-Stokes equations Indiana Univ. Math. J. 42 775-789

[11] Constantin P, Fefferman C and Majda A 1996 Geometric constraints on potentially singular solutions for the 3D Euler equations Commun. Partial Diff. Eqns 21 559-571

[12] Deng J, Hou T and Yu X 2005 Geometric properties and nonblowup of 3D incompressible Euler flow Commun. Partial Diff. Eqns 30 225-243

[13] Deng J, Hou T and Yu X 2006 Improved geometric conditions for non-blowup of the 3D incompressible Euler equation Commun. Partial Diff. Eqns 31 293-306

[14] Escauriaza L, Seregin G A and Sverák V $2003 L_{3, \infty}$-solutions of Navier-Stokes equations and backward uniqueness Uspekhi Mat. Nauk. 58 3-44

[15] FAN J, JiAng S, NAKARUMa G AND ZhOU Y 2011 Logarithmically improved regularity criteria for the NavierStokes and MHD equations J. Math. Fluid Mech. 13 557-571

[16] Giga Y 1986 Solutions for semilinear parabolic equations in $L^{p}$ and regularity of weak solutions of the NavierStokes equations J. Diff. Eqns 62 186-212

[17] Gruji Z and Zhang Q S 2006 Space-time localization of a class of geometric criteria for preventing blow-up in the 3D NSE Commun. Math. Phys. 262 555-564

[18] Kato T and Ponce G 1988 Commutator estimates and the Euler and Navier-Stokes equations Commun. Pure Appl. Math. 41 891-907

[19] Katz N AND PAVLOVIC N 2002 A cheap Caffarelli-Kohn-Nirenberg inequality for the Navier-Stokes equation with hyper-dissipation Geom. Funct. Anal. 12 355-379

[20] Kukavica I AND Ziane M 2006 One component regularity for the Navier-Stokes equations Nonlinearity 19 453-469

[21] Kukavica I and Ziane M 2007 Navier-Stokes equations with regularity in one direction J. Math. Phys. 48 065203

[22] Ladyzhenskaya O A 1967 On uniqueness and smoothness of generalized solutions to the Navier-Stokes equations Zapiski Nauchn. Seminar POMI 5 169-185

[23] LeE J 2012 Notes on the geometric regularity criterion of 3D Navier-Stokes system J. Math. Phys. 53073103

[24] Prodi G 1959 Un teorema di unicità per el equazioni di Navier-Stokes Ann. Mat. Pura Appl. 48 173-182 
[25] Seregin G And Sverák V 2002 Navier-Stokes equations with lower bounds on the pressure Arch. Ration. Mech. Anal. 163 65-86

[26] SerRin J 1962 On the interior regularity of weak solutions of the Navier-Stokes equations Arch. Ration. Mech. Anal. 9 187-195

[27] Struwe M 1988 On partial regularity results for the Navier-Stokes equations Commun. Pure and Appl. Math. 41 437-458

[28] TAO T 2009 Global regularity for a logarithmically supercritical hyperdissipative Navier-Stokes equation Anal. Partial Diff. Eqns 2 361-366

[29] Tran C V and Yu X 2015 Depletion of nonlinearity in the pressure force driving Navier-Stokes flows Nonlinearity 28 1295-1306

[30] Tran C V and Yu X 2016 Regularity of Navier-Stokes flows with bounds for the pressure J. Math. Fluid Mech. submitted

[31] VASSEuR A 2009 Regularity criterion for 3D Navier-Stokes equations in terms of the direction of the velocity Appl. Math. 54 47-52.

[32] ZHOU Y 2002 A new regularity criterion for the Navier-Stokes equations in terms of the gradient of one velocity component Methods Appl. Anal. 9 563-578

[33] Zhou Y 2005 On regularity criteria in terms of pressure for the Navier-Stokes equations in $\boldsymbol{R}^{3}$ Proc. Amer. Math. Soc. 134 149-156

[34] ZHоU Y 2005 A new regularity criterion for the Navier-Stokes equations in terms of the vorticity direction Monatsh. Math. 144 251-257

[35] Zhou Y And Pokorny M 2010 On the regularity of the solutions of the Navier-Stokes equations via one velocity component Nonlinearity 23 1097-1107

Chuong V. Tran: School of Mathematics and Statistics, University of St Andrews, St Andrews KY16 9SS, UNited Kingdom

E-mail address: cvt1@st-andrews.ac.uk

Xinwei Yu: Department of Mathematical and Statistical Sciences, University of Alberta, Edmonton, AB, T6G 2G1, CANADA

E-mail address: xinweiyu@math.ualberta.ca 